\title{
Positive Predictive Value (PPV)
}

National Cancer Institute ( $\mathrm{NCl})$

\section{Source}

National Cancer Institute (NCI). Positive predictive value.

The likelihood that an individual with a positive test result truly has the particular gene and/or disease in question. Also called PPV. 